\title{
Gold nanoparticle liquid crystal composites as a tunable nonlinear medium
}

\author{
A. Acreman,,$^{1, *}$ M. Kaczmarek, ${ }^{1}$ and G. D’Alessandro ${ }^{2}$ \\ ${ }^{1}$ Physics and Astronomy, University of Southampton, Southampton, England, United Kingdom \\ ${ }^{2}$ Mathematical Sciences, University of Southampton, Southampton, England, United Kingdom
}

(Received 29 April 2014; published 8 July 2014)

\begin{abstract}
We investigate the nonlinearity of a liquid crystal cell doped with gold nanoparticles by considering their selective absorption. Such nonlinearities are promising for optical processing applications and optical limiters. Systems displaying thermal nonlinearities are particularly attractive as the maximum nonlinearity may occur in the absence of an applied field and additionally this nonlinearity can be controlled by the reorientation of the liquid crystal. We show that there exists a theoretical optimum concentration of absorbers, which maximizes the nonlinearity. Further we show that the nonlinearity of the system can be tuned by the reorientation of the liquid crystal host, with the nonlinearity decreasing from $9 \times 10^{-5} \mathrm{~cm}^{2} \mathrm{~W}^{-1}$ to zero by the application of a magnetic field of the order of 0.01 Tesla. This allows a fine control of the diffraction efficiency and, in principle, many other nonlinear effects.
\end{abstract}

DOI: 10.1103/PhysRevE.90.012504

\section{INTRODUCTION}

The doping of liquid crystals (LCs) with nanoparticles has become a common method of improving their optical, magnetic, electrical and physical properties [1]. For example, ferroelectric nanoparticles have been shown to decrease the Fréedericksz threshold and increase the birefringence of nematics [2,3]. Similarly, ferromagnetic nanoparticles have been used to increase the sensitivity of the host liquid crystals to an applied magnetic field $[4,5]$.

Gold nanoparticles have also been investigated as possible dopants for liquid crystals. Initially this was due to their potential to form a tunable, self-organizing, three-dimensional (3D) metamaterial suitable for the visible and infrared regions of the optical spectrum [6]. However gold nanoparticles have since also been shown to improve the electro-optical properties of their host liquid crystals. Such suspensions display increased dielectric anisotropy and birefringence, lowered optical and electrical Fréedericksz thresholds, and increased thermal stability [7-12]. Moreover, even low concentration suspensions have increased the nonlinearity of hybrid photorefractive liquid crystal cells [13]. Liquid crystals have also been shown to cause gold nanoparticles to spontaneously form linear self-assemblies [14] and large 3D plasmonic crystals have been formed in an LC host using optical tweezers [15]. Gold nanoparticles functionalized with liquid crystalline surfactants have been demonstrated to form large, complex, self-assembled structures, which exhibit anisotropic absorption [16,17]. Furthermore these samples also show pseudo-liquid-crystal phases, with mesogenic functionalized gold nanorods showing a magnetically controllable nematic phase [18-20]. Finally gold nanoparticle liquid crystal suspensions have been shown to exhibit large thermal nonlinearities with $n_{2}$ coefficients of up to $1.9 \times 10^{-5} \mathrm{~cm}^{2} \mathrm{~W}^{-1}$ [21].

Gold nanoparticles are of particular interest due to their plasmon resonances, which occur in the optical spectrum $[22,23]$. We are specifically interested in the decay of these plasmons, which leads to heating of the surrounding

*Corresponding author: A.Acreman@soton.ac.uk
PACS number(s): 42.70.Df, 42.25.Bs, 42.79.Dj, 78.15.+e

material, an effect that has already been investigated as a potential cancer treatment [24]. In this paper we focus on the large thermal nonlinearity induced by the local heating of gold nanoparticle suspensions in a liquid crystal [21,25]. While other absorbing systems such as dye-doped liquid crystals can exhibit giant nonlinearities of up to $1000 \mathrm{~cm}^{2} \mathrm{~W}^{-1}$, these require the application of a bias field. Gold nanoparticles are preferable to dyes as absorbers for several reasons: their absorption cross section is greater by over an order of magnitude and, due to their excellent thermal stability, they do not bleach [22].

The nonlinearity of gold nanoparticle liquid crystal composites has been investigated experimentally and theoretically by Ouskova et al. with an aim towards optical processing applications [21]. They doped a nematic liquid crystal with small gold nanospheres in order to enhance its thermal nonlinearity. Figure 1 shows a schematic diagram of their experimental setup. Two pump beams write a refractive index grating on the liquid crystal cell [21], which is probed by a third beam. They also modeled the thermal nonlinearity by solving the thermal diffusion equation. The best theoretical estimates they obtain are larger than the experimental values [26]. In particular, the overshoot is larger in the system with greater absorbance of the light beams [21]. In order to explain this difference we extend here their model to include the effect of pump and probe absorbance.

In the following section we find the mathematical form of the temperature distribution within such a LC system by solving the heat diffusion equation for an absorbing material. By comparing our results to experimental data in Ref. [21] we show that the consideration of the attenuation of the beams is critical to accurately modeling the system. In Sec. III A we examine how the thermal nonlinearity varies with the concentration of absorbing particles and show that customization of the system can be improved by using gold nanorods as absorbers. In Sec. III B we investigate the control of the nonlinearities for both gold nanorod and nanosphere suspensions by a magnetic field. This was done by solving for the director profile considering the thermal change in the elastic constants and diamagnetic anisotropy. We investigate and compare the different components of the nonlinearity 


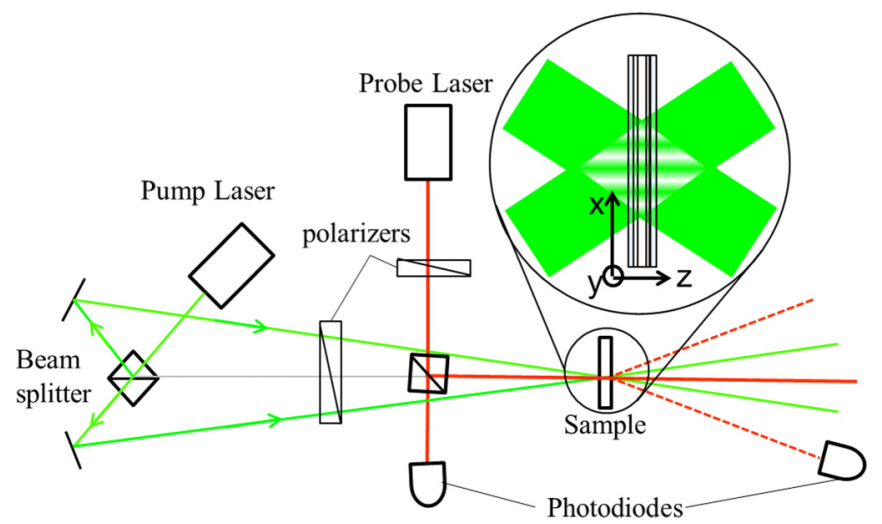

FIG. 1. (Color online) Schematic diagram of the diffraction efficiency experimental setup used in Ref. [21] to measure the thermal nonlinearity of the liquid crystal gold nanoparticle suspensions. In the configuration considered in this paper the polarizations of the pump and probe beams are parallel to the liquid crystal director which is in the $\hat{\boldsymbol{y}}$ direction.

associated with separate coupled physical effects caused by the reorientation of the liquid crystal. We then compare the complete nonlinearity of gold nanorod and nanosphere suspensions discussing the advantages of each. Finally we discuss the conclusions of our work and the potential applications of such nonlinear systems.

\section{HEATING MODEL AND COMPARISON TO EXPERIMENT}

Our model of the thermal nonlinearity is an extension of the one developed by Ouskova et al. in Ref. [21]. We assume that the liquid crystal is heated by pointwise absorption of light by uniformly dispersed gold nanoparticles. We neglect any liquid crystal absorption and thermally driven fluid motion. However, contrary to Ouskova et al., we do not work in the undepleted pump approximation. As shown in Ref. [21] the concentrations required to achieve observable thermal nonlinearity can lead to significant attenuation through the cell with a decrease in the pump intensity of up to $90 \%$. We will show later in this section that accounting for this absorption significantly changes the predicted thermal nonlinearity of the doped liquid crystal and, in fact, gives remarkably good agreement with the experimental data of Ref. [21].

The intensity $I(z, \lambda)$ of a optical beam of a wavelength $\lambda$ propagating a distance $z$ through an absorbing material is given by the the Beer-Lambert law [27],

$$
I(z, \lambda)=I_{0} e^{-\int_{0}^{z} \alpha\left(z^{\prime}, \lambda\right) d z^{\prime}},
$$

where $I_{0}$ is the initial intensity of the beam and $\alpha(z, \lambda)$ is the spatially dependent absorption coefficient at the wavelength $\lambda$. This absorption leads to a temperature change $\Delta T$ within the system. In the geometry considered here, shown in Fig. 1, only diffusion along the ordinary optical axis needs to be considered [26]. Consequently the temperature can be found by solving the heat diffusion equation at equilibrium,

$$
K_{l c} \nabla^{2} \Delta T=\frac{\alpha_{p}(z) I_{\text {pump }}(z)}{2}[1+\cos (q x)] .
$$

Here $K_{l c}$ is the thermal conductivity of the liquid crystal along the ordinary optical axis, $q=2 \pi / \Lambda$ is the wave number of the grating for a grating spacing $\Lambda, \alpha_{p}$ is the absorption of the material at the wavelength of the pump beams. $I_{\text {pump }}$ is the combined intensity of the pump beams throughout the cell and is attenuated as shown in Eq. (1). Finally $x$ and $z$ are distances along the intensity grating and into the cell respectively as indicated in Fig. 1. $\Delta T$ can be separated into the periodically modulated component $\Delta T_{p}$ and the nonperiodic component $\Delta T_{0}$,

$$
\Delta T(x, z)=\Delta T_{0}(z)+\Delta T_{p}(z) \cos (q x) .
$$

As the thermal conductivity of the glass windows is an order of magnitude larger than that of the liquid crystal we have assumed the temperature variation at the liquid crystal glass interface is zero [21], i.e., $\Delta T_{p}=\Delta T_{0}=0$ at $z=0, L$, where $L$ is the thickness of the cell. Solving Eq. (2) gives

$$
\Delta T_{p}(z)=\frac{\sinh (q z)}{\sinh (q L)} F(L)-F(z),
$$

and

$$
\Delta T_{0}(z)=\left(1-\frac{z}{L}\right) G(z)
$$

where

$$
\begin{aligned}
& F(z)=\frac{1}{q} \int_{0}^{z} \sinh \left[q\left(z-z^{\prime}\right)\right] A\left(z^{\prime}\right) d z^{\prime} \\
& G(z)=\int_{0}^{z} \int_{0}^{z^{\prime}} A\left(z^{\prime \prime}\right) d z^{\prime \prime} d z^{\prime}
\end{aligned}
$$

and $A(z)$ is given by

$$
A(z)=\frac{I_{\mathrm{pump}} \alpha_{p}(z)}{2 K_{l c}} e^{-\int_{0}^{z} \alpha_{p}\left(z^{\prime}\right) d z^{\prime}} .
$$

For homogeneous absorption profiles, $\alpha_{p}(z)=\bar{\alpha}_{p}$, Eqs. (6) and (7) simplify to

$$
F(z)=\frac{I_{\mathrm{pump}} \bar{\alpha}_{p}\left[\bar{\alpha}_{p} \sinh (q z)-q \cosh (q z)+q e^{-\bar{\alpha}_{p} z}\right]}{2 K_{l c} q\left(\bar{\alpha}_{p}^{2}-q^{2}\right)}
$$

and

$$
G(z)=\frac{I_{\text {pump }}}{2 K_{l c} \bar{\alpha}_{p}}\left(\bar{\alpha}_{p} z+e^{-\bar{\alpha}_{p} z}-1\right),
$$

respectively.

The temperature distribution leads to a periodic modulation of the refractive index grating. The effective refractive of a liquid crystal is given by [28]

$$
n(T, \theta)=\frac{n_{e}(T) n_{o}(T)}{\sqrt{n_{e}(T)^{2} \sin ^{2}(\theta)+n_{o}(T)^{2} \cos ^{2}(\theta)}},
$$

where $\theta$ is the angle between the polarization of the probe beam (parallel to the $y$ axis) and the liquid crystal principal optical axis, and $n_{e}(T), n_{o}(T)$ are the temperature-dependent extraordinary and ordinary refractive indices respectively. The devices we consider here operate in the nematic phase of the liquid crystal, where the temperature dependence of the refractive indices can be modeled using an extended Cauchy 
TABLE I. A summary of the parameter values used for the modeling work. These values are used for all the figures except where indicated.

\begin{tabular}{lc}
\hline \hline Parameter & Value $($ Units $)$ \\
\hline$I_{\text {pump }}$ & $33.5\left(\mathrm{Wcm}^{-2}\right)$ \\
$\lambda_{\text {pump }}$ & $532(\mathrm{~nm})$ \\
$\lambda_{\text {probe }}$ & $633(\mathrm{~nm})$ \\
$\alpha_{p}$ & $400\left(\mathrm{~cm}^{-1}\right)$ \\
$\Lambda$ & $50(\mu \mathrm{m})$ \\
$L$ & $55(\mu \mathrm{m})$ \\
$K_{l c}$ & $0.15\left(\mathrm{WK}^{-1} \mathrm{~m}^{-1}\right)$ \\
$T^{\dagger}$ & $326(\mathrm{~K})$ \\
$\gamma$ & 0.2435 \\
$\Delta \chi_{0}$ & $2.52 \times 10^{-7}$ \\
$K_{0}$ & $19.25(\mathrm{pN})$ \\
$\Delta n_{0}$ & 0.211 \\
$A$ & 1.7487 \\
$B$ & $4.896 \times 10^{-4}$ \\
$T_{a}$ & $23\left({ }^{\circ} \mathrm{C}\right)$ \\
Sphere radius & $5(\mathrm{~nm})$ \\
Nanorod minor axis & $10(\mathrm{~nm})$ \\
Nanorod aspect ratio $R$ & 2.5 \\
\hline \hline
\end{tabular}

model $[29,30]$

$$
\begin{aligned}
& n_{e}(T)=A-B T+\frac{2 \Delta n_{0}}{3} S(T), \\
& n_{o}(T)=A-B T-\frac{\Delta n_{0}}{3} S(T),
\end{aligned}
$$

where $A, B$ and $\Delta n_{0}$ are wavelength-dependent fitting parameters and $S(T)$ is the liquid crystal scalar order parameter. We estimate its value using Haller's approximation [31],

$$
S(T)=\left(1-\frac{T}{T^{\dagger}}\right)^{\gamma},
$$

where $T^{\dagger}$ and $\gamma$ are also fitting parameters. In general, $T^{\dagger}$ is slightly higher than the clearing temperature. We have determined all the parameters in Eqs. (10)-(12) by fitting literature E7 refractive index data [30]. Their values at $\lambda_{\text {pump }}$ are reported Table I. Finally, as $T$ in Eq. (12) is the absolute temperature whereas Eq. (4) calculates the change in temperature relative to the background, we have assumed that the system is at an ambient temperature $T_{a}=23^{\circ} \mathrm{C}$. As numerical simulations have shown that the refractive index grating is in phase with the intensity grating we can express the amplitude of the refractive grating $\Delta n$ as

$$
\Delta n=\frac{2}{L \Lambda} \int_{0}^{L} \int_{0}^{\Lambda} n(T, 0) \cos (q x) d x d z .
$$

As the grating spacings considered in this paper are larger than $10 \mu \mathrm{m}$ we can assume the grating thickness to be small with respect to its pitch. In this case, known as the the RamanNath regime, it is possible to obtain an analytical expression for the amplitude of the diffracted orders in terms of Bessel functions [32]. Consequently the diffraction efficiency $\eta$, i.e., the ratio of the intensities of the first diffracted order and the

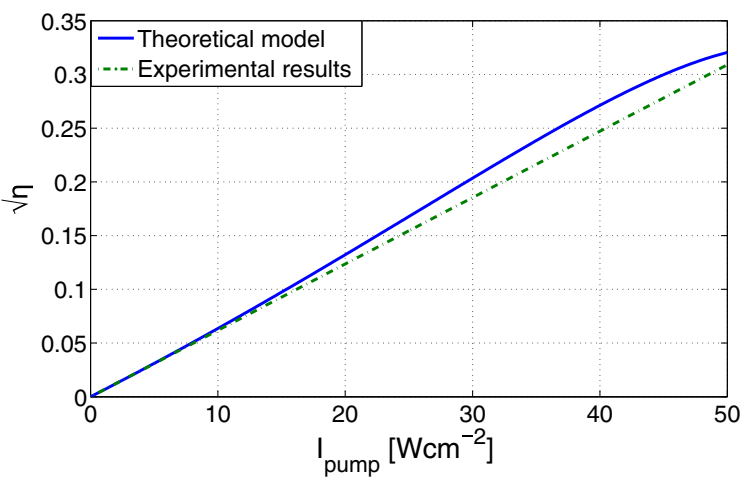

FIG. 2. (Color online) A plot of the theoretical and experimental values for the diffraction efficiency for a range of intensities. The modeling parameters are as listed in Table I with the exception of of the pump and probe absorption coefficients which have values of $390 \mathrm{~cm}^{-1}$ and $276 \mathrm{~cm}^{-1}$ respectively. The experimental curve is the line of best fit from Ref. [21].

probe beam, is given by

$$
\eta=J_{1}^{2}\left(\frac{2 \pi L \Delta n}{\lambda_{\text {probe }}}\right) e^{-\int_{0}^{z} \alpha_{\text {probe }}\left(z^{\prime}\right) d z^{\prime}},
$$

which is an adaptation of the standard expression for diffraction efficiency from a thin sinusoidal grating [32]. The factor $e^{-\int_{0}^{z} \alpha_{\text {probe }}\left(z^{\prime}\right) d z^{\prime}}$ has been included to compensate for the attenuation of the probe beam as it passes through the cell. $J_{1}$ is the first-order Bessel function, $\lambda_{\text {probe }}$ is the wavelength of the probe beam and $\alpha_{\text {probe }}$ is the absorption of the medium at the wavelength of the probe.

In order to validate Eqs. (4)-(14) we have reproduced the experimental data from Ref. [21] using the configuration in Fig. 1. The parameters were chosen to match those in Ref. [21], specifically the probe at a wavelength of $633 \mathrm{~nm}$, a $55 \mu \mathrm{m}$ cell thickness and a $50 \mu \mathrm{m}$ grating spacing. The writing beams have a combined intensity of $33.5 \mathrm{~W} \mathrm{~cm}^{-2}$. The absorption coefficient at pump and probe wavelengths are $390 \mathrm{~cm}^{-1}$ and 276 $\mathrm{cm}^{-1}$, respectively, the wavelengths are specified in Table I.

Figures 2 and 3 show the variation of the diffraction efficiency with intensity and grating spacing as predicted by Eq. (14) with the line of best fit to the experimental data from Ref. [21]. Both plots show excellent agreement. This verifies that our model can be used to accurately predict the diffraction efficiency of a gold nanoparticle liquid crystal cell. The theoretical model used in Ref. [21] neglected the attenuation of both the probe and the pump. The agreement between the experiment results and the model presented here is significantly better, implying that the consideration of the attenuation of the beams is critical to achieving a good agreement.

\section{CONTROLLING THE NONLINEARITY}

\section{A. Optimization of the gold nanoparticle liquid crystal composite}

In the previous section we showed that our model can accurately reproduce the thermal nonlinearity of liquid crystal gold nanoparticle suspensions. In this section we investigate two key issues relevant to the optimization of the system. The 


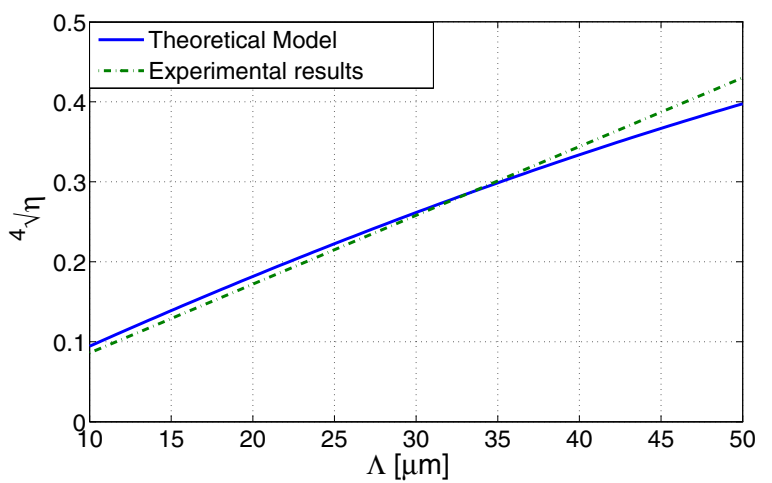

FIG. 3. (Color online) A plot of the theoretical and experimental values for the diffraction efficiency for a range of grating spacings. The modeling parameters are as listed in Table I with the exception of of the pump and probe absorption coefficients which have values of $390 \mathrm{~cm}^{-1}$ and $276 \mathrm{~cm}^{-1}$ respectively. The experimental curve is the line of best fit from Ref. [21].

first of these is the variation of the nonlinearity with respect to the absorption coefficient of the system $\alpha$. This is important as the absorption coefficient is related to the molar concentration of nanoparticles $C$ by

$$
C=\frac{\alpha}{\epsilon}
$$

where $\epsilon$ is the molar extinction coefficient. Consequently understanding how the nonlinearity changes with $\alpha$ allows the selection of an appropriate concentration of nanoparticles for the composite. The second issue is the optimization of the absorption characteristics of the nanoparticles themselves. As the absorption properties of gold nanorods are strongly dependent on their aspect ratio, we will investigate how this can be used to specifically design a system to suit a range of pump and probe wavelengths.

First, however, we need a suitable measure of the nonlinearity. As the heating of the liquid crystal is approximately linearly proportional to the intensity of the pump beams $I_{\text {pump }}$ for heating effects of the order of $\Delta T \approx 10^{\circ} \mathrm{C}$, it is appropriate to define an effective nonlinear refractive index as

$$
n_{2}=\frac{\Delta n}{I_{\text {pump }}},
$$

where $\Delta n$ is the refractive index variation given by Eq. (13). Note that $n_{2}$ is, through Eqs. (13) and (9), a function of $\Lambda, L$, $\alpha$ and $\theta$.

In Fig. 4 we have plotted the nonlinear coefficient against the absorption coefficient of the system at a fixed grating spacing of $50 \mu \mathrm{m}$. The largest effect is seen at $\alpha_{p} \approx 600 \mathrm{~cm}^{-1}$ showing that there is a concentration of particles, which maximizes the nonlinearity. The magnitude of the nonlinearity declines past this point due to the attenuation of the probe beam skewing the temperature distribution toward the initial cell wall. This acts to limit the magnitude of the periodic temperature distribution and consequently the nonlinearity. The location of this peak nonlinearity is also dependent on the thickness of the cell and the grating spacing, with thicker gratings and cells causing the maximum to occur at smaller values of $\alpha$.

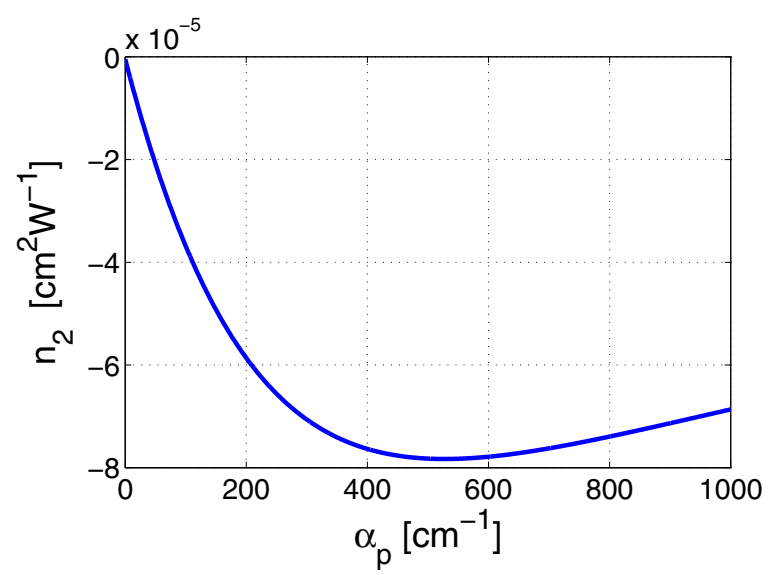

FIG. 4. (Color online) A plot of the nonlinear coefficient against the absorption coefficient at the pump wavelength. With the exception of $\alpha_{p}$ the modeling parameters are listed in Table I.

This absorption coefficient can be converted to a concentration by using the corresponding molar absorption coefficients and Eq. (15). We have calculated that $600 \mathrm{~cm}^{-1}$ converts to approximate concentrations of $1.5 \%, 0.75 \%$, and $0.054 \%$ by weight for $1 \mathrm{~nm}$ and $5 \mathrm{~nm}$ gold spheres and 10 by $25 \mathrm{~nm}$ gold nanorods respectively. These dimensions were chosen as they correspond to nanoparticles synthesized by the groups of Georg Mehl and Torsten Hegmann, which have been specifically designed to be soluble in a room temperature nematics $[8,18,20]$.

While Fig. 4 shows that it is possible to increase the nonlinearity by increasing the concentration of absorbers, this will also increase the attenuation of the probe beam and hence decrease the effective diffraction efficiency. It is worth noting that the attenuation of the probe led to a decrease in the diffraction efficiency by a factor of four in Figs. 2 and 3, as $e^{-\int_{0}^{z} \alpha_{\text {probe }}\left(z^{\prime}\right) d z^{\prime}} \approx 0.25$. Because of this, it is imperative to select nanoparticles, which have minimal absorption at the probe wavelength. Nanorods can be used for this as the wavelength of their long axis plasmon resonance is strongly dependent on their aspect ratio. Consequently they can be specifically synthesized in order to minimize the absorption of the probe.

Figure 5 shows the diffraction efficiency $\eta$ of a suspension of gold nanorods for a range of probe wavelengths and aspect ratios. The absorption properties of the suspensions were modeled using the dipole approximation neglecting the birefringence of the liquid crystal [33]. The dielectric properties of gold were modeled using the Drude model with adaptations to account for the interband transitions and finite size of the particles (see Appendix) [34,35]. The wavelength of the pump beams was set to the absorption peak of the gold nanorods in order to maximize the heating effect. We have chosen a concentration such that $\alpha_{p}=400 \mathrm{~cm}^{-1}$ : this is the largest value of $\alpha_{p}$ that does not lead to significant diffraction into higher orders and is close to the experimental parameters in Ref. [21].

The first observation that can be made from Fig. 5 is that in order to have significant diffraction of the probe beam it is essential to chose a probe wavelength away from the plasmon resonance of the particles (dashed line in Fig. 5). Secondly, the diffraction efficiency decreases for longer wavelengths as expected from the inverse proportionality of Eq. (14). Finally, 


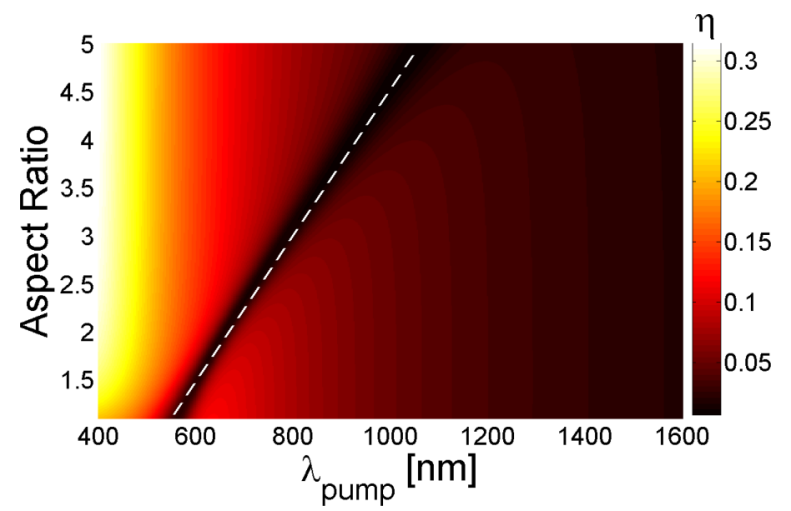

FIG. 5. (Color online) A plot of the diffraction efficiency of a gold nanorod suspension with varying aspect ratios and for a range of probe wavelengths. With the exception of $\lambda_{\text {probe }}$ and the aspect ratio, the modeling parameters are listed in Table I.

the diffraction is fairly insensitive to the aspect ratio of the particles. For the monodispersed samples considered here, the plasmon resonance is very narrow for sufficiently large aspect ratios and, therefore, the absorption away from the resonance is negligible.

\section{B. Controlling the nonlinearity with a magnetic field}

The ordinary refractive index of E7 is three orders of magnitude less sensitive to temperature changes than the extraordinary refractive index $[21,30]$. This difference opens the possibility of actively controlling the nonlinearity of the gold nanoparticle suspension. So far we have treated the case where the incoming probe beam is polarized parallel to the director orientation and, consequently, it observes the largest possible nonlinear refractive index. However, if we were to reorient the liquid crystal so that the beam polarization is aligned with the ordinary refractive index, the resulting nonlinear coefficient should be three orders of magnitude smaller. In principle either an electric or a magnetic field could be used to achieve this change of direction, and consequent modulation of $n_{2}$. Here, for simplicity, we model the effect of a magnetic field. In Sec. IV we will discuss briefly the case of the electric field.

The equilibrium configuration of the liquid crystal director field in a twist-free cell is the solution of

$$
\nabla \cdot[K(T) \nabla \theta]+H^{2} \Delta \chi(T) \sin (2 \theta)=0,
$$

where we have assumed that the liquid crystal lies in the $(y, z)$ plane and that its director forms an angle $\theta$ with the $y$ axis. Here $H$ is the magnetic field applied to the cell, and $\Delta \chi(T)$ and $K(T)$ are the temperature-dependent diamagnetic anisotropy and splay elastic constant, respectively. These can be approximated by $[36,37]$,

$$
\Delta \chi(T)=\Delta \chi_{0} S(T), \quad K(T)=K_{0} S^{2}(T),
$$

where $\Delta \chi_{0}$ and $K_{0}$ are fitting parameters the values of which are shown in Table I. For $S(T)$ we have used the function (12) fitted to the birefringence data and estimated $\Delta \chi_{0}$ and $K_{0}$ using room temperature values of $\Delta \chi$ and $K$. We have solved numerically Eq. (17) using the coefficient form differential equation solver in COMSOL MULTIPHYSICS ${ }^{\circledR}$. As we are not interested in a detailed quantitative analysis of the nonlinear coefficient as a function of the magnetic field, we have neglected the effect that the change in orientation of the liquid crystal has on the heat diffusion. This effect is relatively small and does not change qualitatively the features that we analyze here.

From Eqs. (9) and (17) we can see that the magneticfield-induced reorientation of the liquid crystal will affect its thermal nonlinearity through three factors: (F1) the change in the particle absorption, $\alpha$; (F2) the change in the observed refractive index; and (F3) the thermal dependence of $\theta$ through $K(T)$ and $\Delta \chi(T)$. We now consider these three factors in turn.

F1: Changes to the absorption. As the liquid crystal reorients, the refractive index surrounding the nanosphere changes according to Eq. (9). This shifts the plasmon wavelength out of resonance with the pump and, hence, reduces the particle absorption coefficient [33]. This can be expressed as

$$
\alpha(\theta)=\alpha_{n_{e}} \cos ^{2}(\theta)+\alpha_{n_{o}} \sin ^{2}(\theta),
$$

where $\alpha_{n_{e}}$ and $\alpha_{n_{o}}$ are the absorption assuming the surrounding refractive index is equal to $n_{e}$ and $n_{o}$ respectively. For a suspension of gold nanospheres of $5 \mathrm{~nm}$ radius in the liquid crystal E7 $\alpha_{n_{e}} / \alpha_{n_{o}}=1.42$, i.e., reorientation reduces the absorption by a factor of 1.42 .

The change in absorption is enhanced for nanorods: these are expected to reorient with the liquid crystal thus changing the excited plasmon resonance. When $\theta=0$ the plasmon is excited along the long axis of the rod, but as the rod reorients and $\theta \rightarrow \pi / 2$ the plasmon is instead excited along the short axis. This reduces the absorption of the rods at the wavelength of the long axis plasmon resonance by over three orders of magnitude [33,38]. The rod absorption coefficient can be expressed as

$$
\alpha(\theta)=\alpha_{l} \cos ^{2}(\theta)+\alpha_{s} \sin ^{2}(\theta),
$$

where $\alpha_{l}$ and $\alpha_{s}$ are the absorption due to the plasmon oscillations along the of the long and short axis of the rod respectively. At the wavelength of the long axis plasmon resonance $\alpha_{l} / \alpha_{s}=10^{-3}$.

The effect of reorientation on absorption is illustrated in Fig. 6 for typical nanoparticle sizes (see Table I). From the figure it is clear that the absorption decreases with the applied

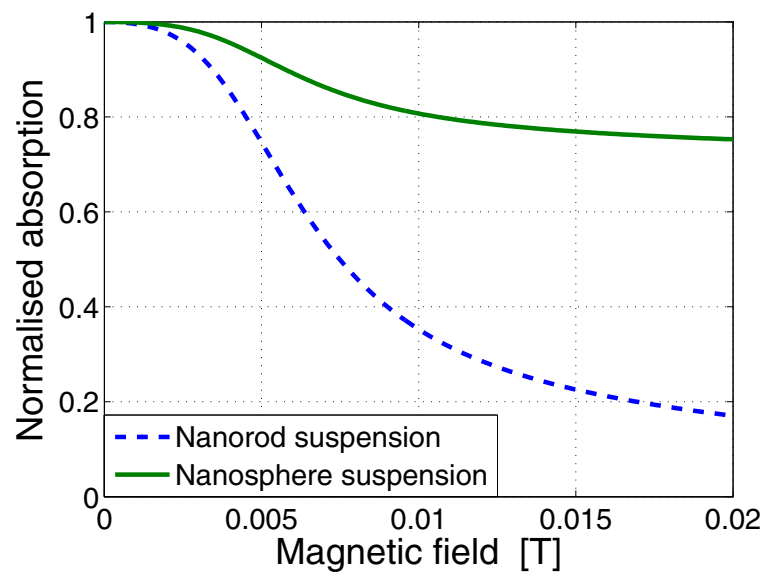

FIG. 6. (Color online) A plot of the magnetic field dependent absorption of a liquid crystal cell doped with gold nanospheres and gold nanorods. All parameters as listed in Table I. 


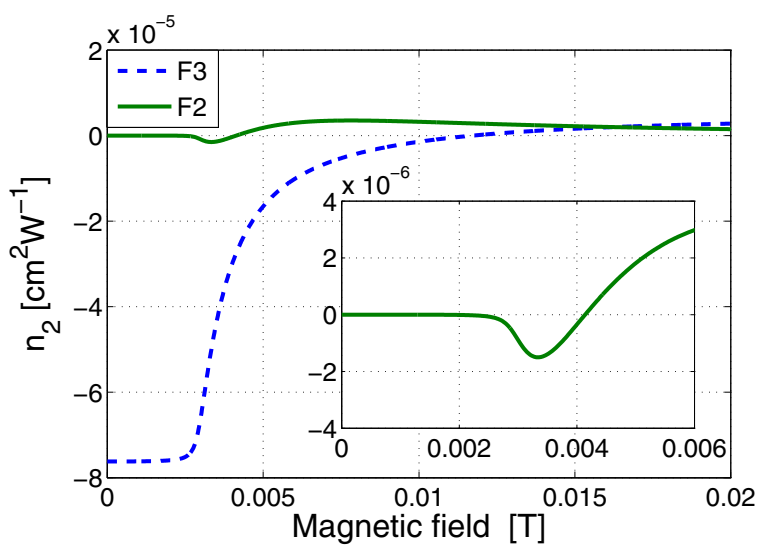

FIG. 7. (Color online) Plot of the effects that the magnetic field induced reorientation has on $n_{2}$. F2: Effect on birefringence. The dashed blue curve is the plot of $n_{2}$ computed assuming that $T=$ $T(x, z)$ in Eq. (9), but $T=T_{a}$ in Eq. (17). F3: Effect of the director parameters. The solid green curve is the plot of $n_{2}$ computed assuming that $T=T_{a}$ in Eq. (9), but $T=T(x, z)$ in Eq. (17). All parameters as listed in Table I. The inset shows a magnification of the effect of the director parameters (F3) highlighting the sign change of the nonlinearity.

magnetic field. As expected, the decrease is considerably larger for nanorods than for spheres, but is limited by regions near the cell walls, which do not reorient.

F2: Temperature sensitivity of the birefringence. This effect is illustrated by the blue curve in Fig. 7. As the liquid crystal reorients the effective refractive index seen by the probe becomes closer and closer to the ordinary refractive index of the liquid crystal. This is much less sensitive to temperature variations than the extraordinary refractive index and it increases, rather than decreases, with temperature. The net result is that the nonlinearity decreases in magnitude with the applied magnetic field and changes sign at sufficiently high magnetic fields ( 0.012 Tesla in the case of Fig. 7).

F3: Effect of the director parameters. This effect is illustrated by the green curve in Fig. 7. We observe first that at low magnetic fields this effect is approximately 50 times smaller than the birefringence contribution (blue curve). At high magnetic fields the magnitudes of the two contributions become comparable and the nonlinear behavior of the liquid crystal is a combination of both effects. The second observation is that the behavior with the applied field is nonmonotonic, as highlighted in the inset. This is the result of the competing effects of $K(T)$ and $\Delta \chi(T)$. At low magnetic fields the thermal effect on the elastic constant dominates: this increases the reorientation of the liquid crystal in the hot regions, thus giving a negative nonlinear coefficient. At higher magnetic fields the liquid crystal is fully reoriented and $K$ no longer plays a significant role. Consequently the thermal change in $\Delta \chi(T)$ dominates and the situation is reversed: hotter regions have smaller diamagnetic anisotropy and, thus, reorient less, leading to a change of sign of the nonlinearity.

Combined effects. The effects of all these factors are combined in Fig. 8. A quick comparison with Fig. 7 shows immediately that for both spheres and nanorods the behavior of the nonlinearity with the applied magnetic field is dominated by F2, its effect on the birefringence.

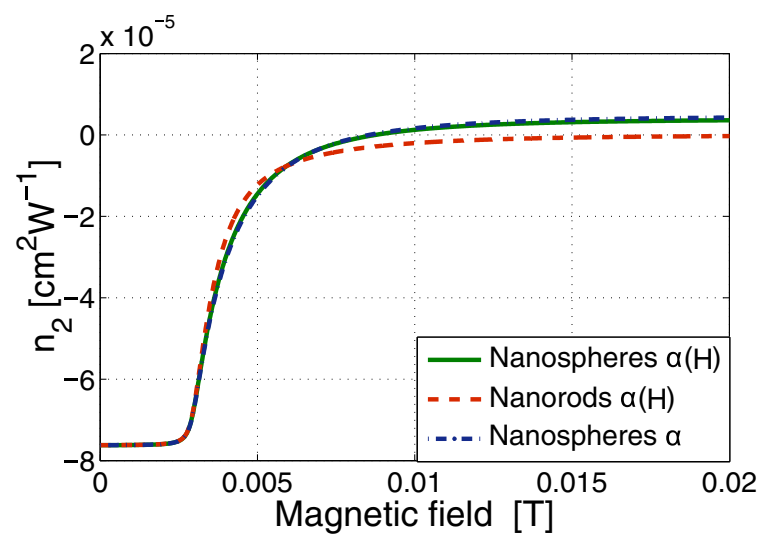

FIG. 8. (Color online) Plot of the nonlinear coefficient $n_{2}$ against the applied magnetic field for: the gold nanorod suspension (dashed red line), the nanosphere suspension (solid green line) including factors F1-F3 and, for comparison, the nanosphere suspension neglecting F1 (dashed and dotted blue line). In the latter case $\alpha$ is no longer a function of the applied magnetic field $H$. All parameters as listed in Table I.

In the case of nanospheres the behavior of $n_{2}$ with the applied magnetic field is determined by F2 and F3, while F1 plays only a very minor role (compare the green and blue curves in Fig. 8). In particular, $n_{2}$ decreases with magnetic fields and changes sign as expected from Fig. 7. At this point the material changes from defocusing to focusing, opening up the possibility of temperature and magnetic fields tuning of the sign of the nonlinearity [25]. In the case of nanorods, the effect of the absorption is more significant and consequently at high magnetic fields the nonlinearity tends to zero, rather than change sign.

We conclude this section by remarking that even though the modulation of the absorption with magnetic fields has only a limited effect on $n_{2}$, it has a very large effect on the pump beams. While in nanosphere suspensions the pump beams are always significantly absorbed, they will be only weakly attenuated by nanorod suspensions at sufficiently high magnetic fields.

\section{CONCLUSION}

We have modeled the optical thermal nonlinearity of gold nanoparticle liquid crystal suspensions by extending previous models to include the attenuation of the pump and probe beams. As our model gives a much better fit to existing experimental data we can conclude that the consideration of this attenuation is key to accurately predicting the behavior of the thermal nonlinearity. Due to the attenuation of the beams, the scaling of the induced refractive index grating with the number of absorbers is no longer trivial. Our model predicts a critical concentration of particles above which the magnitude of the nonlinearity will decrease. Specific to the geometry we consider, the peak nonlinearity occurs at $600 \mathrm{~cm}^{-1}$ which equates to concentrations of $1.5 \%, 0.75 \%$, and $0.054 \%$ for $1 \mathrm{~nm}$ and $5 \mathrm{~nm}$ gold spheres and $10 \times 25 \mathrm{~nm}$ gold nanorods, respectively. The disparity in the concentrations is important as it can be extremely difficult to suspend nanoparticles in a liquid crystal host and complex surfactants are usually required. 
Because of this, any decrease in the number of particles required is a boon to those working with the system. We have also shown that the system can be customized for different probe and pump wavelengths by using gold nanorods instead of nanospheres.

In this paper we have assumed that a magnetic field is used to reorient the liquid crystal. It is also possible to use an electric field to this effect. The main difference between the two driving fields are that (i) the electric field has a far higher sensitivity to the alignment of the liquid crystal than the magnetic field, (ii) gold particles affect the material properties of the liquid crystal [7,39], and (iii) the particles may migrate to regions of high field intensity due to dielectrophoretic forces. While these effects may have to be taken into account for precise quantitative modeling of the device tunability under the influence of the electric field, they are all sufficiently small that we do not expect qualitative differences when using either field for controlling the orientation of the liquid crystal.

We also investigated the dependence of the nonlinearity on the reorientation of the liquid crystal host showing that an applied field can be used to dramatically decrease the nonlinearity and even switch the system from a self-focusing to self-defocusing regime. We have identified that the dominant effect is the change of the thermal dependence in the refractive index due to the reorientation of the liquid crystal. While the gold nanosphere suspension displays a slightly greater tunability in the nonlinearity, the nanorod suspension allows the attenuation of the probe beam to be significantly reduced. Consequently, each system lends itself to different applications.

The analysis carried out in this paper assumes that the heating source is a regular grating. However, the effect we have studied is much more general: for example, the differential heating of the liquid crystal could be caused by a spatially inhomogeneous beam. While the thermal nonlinearity is slow, the flexibility of its behavior and the latitude with which it can be controlled may make it ideal for non-time-critical, low-power applications.

\section{ACKNOWLEDGMENTS}

We would like to thank Elena Ouskova and Tim Sluckin for their invaluable suggestions and discussions, and Matthew Proctor for his assistance with this project. We acknowledge the support of COST Action 1205.
TABLE II. The physical meanings and values for the parameters used to model the dielectric properties of gold, Eq. (A2). All values taken from Refs. [34,40], with the exception of $A_{1}$ and $A_{2}$, which were slightly changed to give a better fit for wavelengths between 400 and $600 \mathrm{~nm}$.

\begin{tabular}{llll}
\hline \hline \multirow{2}{*}{$\begin{array}{l}\text { Parameter } \\
\text { (units) }\end{array}$} & \multicolumn{1}{c}{ Description } & \multicolumn{2}{c}{ Value } \\
\cline { 3 - 4 } & & $a=1$ & $a=2$ \\
\hline$A_{a}$ & Unitless transition amplitude & 0.99 & 1.42 \\
$\lambda_{a}(\mathrm{~nm})$ & Interband transaction wavelength & 468 & 331 \\
$\theta_{a}$ & Interband transaction phase & $-\pi / 4$ & $-\pi / 4$ \\
$\gamma_{a}(\mathrm{~nm})$ & Interband transaction dampening & 2300 & 940 \\
$\mu_{a}$ & Order of the pole & 1 & 1 \\
$\gamma_{c}(\mathrm{~nm})$ & Dampening of conduction electrons & \multicolumn{2}{c}{17000} \\
$\lambda_{p}(\mathrm{~nm})$ & Plasmon wavelength & \multicolumn{2}{c}{145} \\
$\epsilon_{\infty}$ & Dielectric constant as $\lambda \rightarrow 0$ & \multicolumn{2}{c}{1.53} \\
$\Xi$ & Scaling parameter & \multicolumn{2}{c}{0.7} \\
\hline \hline
\end{tabular}

\section{APPENDIX: NANOPARTICLE MODEL}

In this Appendix we give some additional details of the model used to determine the absorption spectra of aligned gold nanorods that enabled us to produce Fig. 5. We have assumed that the particles are ellipsoids, coated with a thin layer of surfactant and immersed in an isotropic liquid with refractive index equal to the extraordinary refractive of E7. As the particle size is much smaller than the light wavelength, its interaction with the light field can be considered quasistatic and the scattered field is approximated by an electrostatic dipole (dipole approximation). Finally, we have computed the dielectric permittivity of gold using a Drude model with adaptations to account for the interband transitions and the finite particle size.

The absorption cross section $C_{a b s}$ of aligned nanorods is given by

$$
C_{a b s}=k \Im\left[\cos (\theta)^{2} \alpha_{1}+\sin (\theta)^{2} \alpha_{s}\right],
$$

where $\alpha_{l}, \alpha_{s}$ are the the polarizabilities along their long and short axis respectively, and $\theta$ is the angle between the long axis and the polarization of the incident light. The polarizabilities can be found by solving for the scattered field of an ellipsoidal gold particle coated with a dielectric layer in the dipole approximation [33]:

$$
\alpha_{j}=V \frac{\left(\epsilon_{2}-\epsilon_{m}\right)\left[\epsilon_{2}+\left(\epsilon_{1}-\epsilon_{2}\right)\left(L_{j}^{(1)}-f L_{j}^{(2)}\right)\right]+f \epsilon_{2}\left(\epsilon_{1}-\epsilon_{2}\right)}{\left(\epsilon_{m}+L_{j}^{(2)}\left(\epsilon_{2}-\epsilon_{m}\right)\right)\left[\epsilon_{2}+\left(\epsilon_{1}-\epsilon_{2}\right)\left(L_{j}^{(1)}-f L_{j}^{(2)}\right)\right]+f \epsilon_{2} L_{j}^{(2)}\left(\epsilon_{1}-\epsilon_{2}\right)},
$$

where $L_{j}^{(1)}$ and $L_{j}^{(2)}$ are the geometric functions of the inner and outer ellipsoids [33], $V$ is the total volume of the particle including the surfactant layer, and $f$ is the ratio of the volume of the gold core relative to $V . \epsilon_{\{1,2, m\}}$ are the dielectric permittivities of the gold core, surfactant and surrounding material, respectively. In producing Fig. 5 we have assumed that the surfactant layer has thickness of $1.2 \mathrm{~nm}$ and dielectric permittivity $\epsilon_{2}=2.128$, values that are close to those of dodecanethiol, a common surfactant for gold nanoparticles. For the surrounding material the extraordinary refractive index of E7 was used using the extended Cauchy model for the wavelength dependence. 
The dielectric function of gold was modeled using a Drude model with adaptations for the interband transitions and finite particle size. This is given by [34]

$$
\begin{aligned}
\varepsilon(\lambda)= & \varepsilon_{\infty}-\frac{1}{\lambda_{p}\left(\frac{1}{\lambda^{2}}+\frac{i}{\lambda \gamma_{c, 1}}\right)} \\
& +\sum_{a=1,2} A_{a}\left(\frac{e^{i \theta_{a}}}{\left(\frac{1}{\lambda_{a}}-\frac{1}{\lambda}-\frac{i}{\gamma_{a, 1}}\right)}+\frac{e^{-i \theta_{a}}}{\left(\frac{1}{\lambda_{a}}+\frac{1}{\lambda}+\frac{i}{\gamma_{a, 1}}\right)}\right) .
\end{aligned}
$$

The physical meanings and values for these parameters are given in Table II, with the exception of $\gamma_{c}, \gamma_{1,1}$, and $\gamma_{2,1}$. These are corrections to the dampening lengths of the conduction electrons, $\gamma$, and interband transitions, $\gamma_{\{1,2\}}$ due to the finite size of the particles. They are given by [35]:

$$
\frac{1}{\gamma_{\alpha, 1}}=\frac{1}{\gamma_{\alpha}}+\frac{\Xi v_{f}}{2 \pi c R}, \quad \alpha=\{c, 1,2\},
$$

where $\Xi$ is a scaling parameter, $v_{f}$ is the Fermi velocity, $c$ is the speed of light in a vacuum and $R$ is the adapted mean free path of the electrons, which is equal to the particle size.
[1] T. H. S. Umadevi, G. Venkatchalam, Handbook of Liquid Crystals, 2nd ed. 5 (Wiley, New York, 2014).

[2] M. R. Herrington, O. Buchnev, M. Kaczmarek, and I. Nandhakumar, Mol. Cryst. and Liq. Cryst. 527, 72 (2010).

[3] Y. Reznikov, O. Buchnev, O. Tereshchenko, V. Reshetnyak, A. Glushchenko, and J. West, Appl. Phys. Lett. 82, 1917 (2003).

[4] N. Podoliak, O. Buchnev, O. Buluy, G. D'Alessandro, M. Kaczmarek, Y. Reznikov, and T. J. Sluckin, Soft Matter 7, 4742 (2011).

[5] E. Ouskova, O. Buluy, C. Blanc, H. Dietsch, and A. Mertelj, Mol. Cryst. Liq. Cryst. 525, 104 (2010).

[6] R. Pratibha, K. Park, I. I. Smalyukh, and W. Park, Opt. Express 17, 19459 (2009).

[7] M. Draper, I. M. Saez, S. J. Cowling, P. Gai, B. Heinrich, B. Donnio, D. Guillon, and J. W. Goodby, Adv. Funct. Mater. 21, 1260 (2011).

[8] J. Mirzaei, M. Urbanski, H.-S. Kitzerow, and T. Hegmann, Philos. T. R. Soc. A 371, 20120256 (2013).

[9] H. Qi and T. Hegmann, Liquid Crystals Today 20, 102 (2011).

[10] S. Khatua, P. Manna, W.-S. Chang, A. Tcherniak, E. Friedlander, E. R. Zubarev, and S. Link, J. Phys. Chem. C 114, 7251 (2010).

[11] S. Yabu, Y. Tanaka, K. Tagashira, H. Yoshida, A. Fujii, H. Kikuchi, and M. Ozaki, Opt. Lett. 36, 3578 (2011).

[12] A. Sharma, M. Worden, and T. Hegmann, Ferroelectrics 431, 154 (2012).

[13] N. Podoliak, D. Bartczak, O. Buchnev, A. G. Kanaras, and M. Kaczmarek, J. Phys. Chem. C 116, 12934 (2012).

[14] J. Milette, S. J. Cowling, V. Toader, C. Lavigne, I. M. Saez, R. Bruce Lennox, J. W. Goodby, and L. Reven, Soft Matter 8, 173 (2012).

[15] I. Muševič, M. Škarabot, U. Tkalec, M. Ravnik, and S. Žumer, Science 313, 954 (2006).

[16] J. Dintinger, B.-J. Tang, X. Zeng, F. Liu, T. Kienzler, G. H. Mehl, G. Ungar, C. Rockstuhl, and T. Scharf, Adv. Mater. 25, 1999 (2013).

[17] S. Frein, J. Boudon, M. Vonlanthen, T. Scharf, J. Barber, G. Süss-Fink, T. Bürgi, and R. Deschenaux, Helv. Chim. Acta 91, 2321 (2008).
[18] S. Umadevi, X. Feng, and T. Hegmann, Adv. Func. Mater. 23, 1393 (2013).

[19] J. M. Wolska, D. Pociecha, J. Mieczkowski, and E. Gorecka, Soft Matter 9, 3005 (2013).

[20] L. Cseh and G. Mehl, J. Am. Chem. Soc 128, 13376 (2006).

[21] E. Ouskova, D. Lysenko, S. Ksondzyk, L. Cseh, G. H. Mehl, V. Reshetnyak, and Y. Reznikov, Mol. Cryst. and Liq. Cryst. 545, 123 (2011).

[22] S. Eustis and M. A. El-Sayed, Chem. Soc. Rev. 35, 209 (2006).

[23] M.-C. Daniel and D. Astruc, Chem. Rev. 104, 293 (2004).

[24] D. Bartczak, O. L. Muskens, T. M. Millar, T. Sanchez-Elsner, and A. G. Kanaras, Nano. Lett. 11, 1358 (2011).

[25] I. C. Khoo, Liquid Crystals, Physical Properties and Nonlinear Optical Phenomena (Wiley, New York, 1995).

[26] D. Lysenko, E. Ouskova, S. Ksondzyk, L. Cseh, G. H. Mehl, V. Reshetnyak, and Y. Reznikov, Eur. Phys. J. E. 35, 1 (2012).

[27] A. Beer, Annalen der Physik und Chemie 86, 7888 (1852).

[28] P. G. de Gennes and J. Prost, The Physics of Liquid Crystals. (Clarendon Press, Oxford, 1993).

[29] J. Li and S.-T. Wu, J. Appl. Phys. 95, 896 (2004).

[30] J. Li, C.-H. Wen, S. Gauza, R. Lu, and S.-T. Wu, J. Disp. Technol. 1, 51 (2005).

[31] I. Haller, Prog. Solid State Ch. 10, 103 (1975).

[32] R. J. Collier, C. B. Burkhardt, and L. H. Lin, Optical Holography (Academic Press, Waltham, Massachusetts, 1971).

[33] D. R. Bohren and C. F. Huffman, Absorption and Scattering of Light by Small Particles (Wiley, New York, 1983).

[34] P. G. Etchegoin, E. C. Le Ru, and M. Meyer, J. Chem. Phys. 127 189901 (2007).

[35] W. Haiss, N. T. K. Thanh, J. Aveyard, and D. G. Fernig, Anal. Chem. 79, 4215 (2007).

[36] H. Ishikawa, A. Toda, H. Okada, and H. Onnagawa, Liq. Cryst. 22, 743 (1997).

[37] A. Buka and W. De Jeu, J. Phys. 43, 361 (1982).

[38] S. V. Burylov and Y. L. Raikher, Phys. Lett. A 149, 279 (1990).

[39] A. S. Pandey, R. Dhar, S. Kumar, and R. Dabrowski, Liq. Cryst. 38, 115 (2011).

[40] P. B. Johnson and R.-W. Christy, Phys. Rev. B 6, 4370 (1972). 